\title{
Short communication: Diagnostic accuracy of focused lung ultrasonography (FLUS) as a rapid method for the diagnosis of respiratory disease in dairy calves.
}

\begin{tabular}{|r|l|}
\hline Journal: & Journal of Dairy Science \\
\hline Manuscript ID & JDS.2020-19377.R2 \\
\hline Article Type: & Research \\
\hline Author: & O7-Dec-2020 \\
\hline Complete List of Authors: & $\begin{array}{l}\text { Pravettoni, Davide; Università degli Studi di Milano, Dipartimento di } \\
\text { Medicina Veterinaria } \\
\text { Buczinski, Sébastien; Centre hospitalier universitaire veterinaire, } \\
\text { Département de Sciences Cliniques } \\
\text { Sala, Giulia; Università degli Studi di Milano, Dipartimento di Medicina } \\
\text { Veterinaria } \\
\text { Ferrulli, Vincenzo; Università degli Studi di Milano, Dipartimento di } \\
\text { Medicina Veterinaria } \\
\text { Bianchi, Francesco; Università degli Studi di Milano, Dipartimento di } \\
\text { Medicina Veterinaria } \\
\text { Boccardo, Antonio; Università degli Studi di Milano, Dipartimento di } \\
\text { Medicina Veterinaria }\end{array}$ \\
\hline Key Words: & $\begin{array}{l}\text { Bovine respiratory disease, Calves, Focused ultrasonography, Thoracic } \\
\text { ultrasonography }\end{array}$ \\
\hline & \\
\hline
\end{tabular}

\section{SCHOLARONE Manuscripts}


1 Short communication: Diagnostic accuracy of focused lung ultrasonography (FLUS) as a rapid

2 method for the diagnosis of respiratory disease in dairy calves. D. Pravettoni

3 Bovine respiratory disease complex is a multifactorial disease that causes pneumonia in cattle

4 worldwide. Early and accurate diagnosis is crucial for economic and welfare reasons and to reduce

5 the use of antimicrobials. This study evaluated the performance of focused lung ultrasonography

6 (FLUS) limited to two intercostal spaces per side to indicate lung lesions associated with bovine respiratory disease. The results showed that this technique has satisfactory accuracy in diagnosing pneumonia and can be easily applied to older calves where the forelimb musculature precludes access to the cranial thorax.

\section{FOCUSED LUNG ULTRASONOGRAPHY METHOD IN DAIRY CALVES}

Short communication: Diagnostic accuracy of focused lung ultrasonography (FLUS) as a rapid method for the diagnosis of respiratory disease in dairy calves.

${ }^{1}$ Dipartimento di Medicina Veterinaria, Università degli Studi di Milano, Via dell'Università 6, 26900 Lodi, Italy

18 20épartement de Sciences Cliniques, Faculté de Médecine Vétérinaire, Université de Montréal, 3200 19 rue Sicotte, St-Hyacinthe, J2S 2M2, Québec, Canada

$21 *$ Corresponding author:

22 Giulia Sala, DVM, PhD

23 Department of Veterinary Medicine, University of Milan, Via dell'Università, 6, 26900 Lodi (Italy)

24 Tel: +39-02-50334149

e-mail: giulia.sala1@unimi.it 


\section{ABSTRACT}

This study estimates the accuracy of the focused lung ultrasound (FLUS) compared to systematic thoracic ultrasonography (TUS) as the reference test for diagnosing pneumonia in pre- and postweaned dairy calves. One hundred and thirty-five Holstein Friesian calves, aged between 1 to 6 months were enrolled, which were kept in the same pen with 1 or more animals showing signs of bovine respiratory disease complex (BRDC). One operator performed FLUS on each calf, and then a second blinded operator performed TUS on the same calf. For the FLUS, we only scanned the lung lobes that are most frequently affected during BRDC and which are thus easier to detect, i.e., the caudal aspect of the cranial lobe of the left lung (fifth and fourth left intercostal spaces; ICS), the middle lobe of the right lung (fifth right ICS), and the caudal aspect of the cranial lobe of the right lung (fourth right ICS). Pneumonia was diagnosed when a calf had a minimum of 1 small lobular lung lesion which was at least $1 \mathrm{~cm}$ deep within a normally aerated lobe (TUS score of $\geq 2$ ). Diagnostic accuracy indexes of the FLUS were calculated using TUS as the gold standard. The McNemar test was performed to evaluate the differences between the 2 techniques. In addition, an inter-test agreement was assessed using the weighted kappa test. A total of 76 out of 135 calves had a TUS score of $\geq 2$ and were therefore considered to be affected by BRDC. FLUS had a sensitivity of $81.6 \%$ ( $95 \%$ confidence interval $71.0 \%$ to $89.5 \%)$, specificity $=100 \%$ (95\% confidence interval $93.9 \%$ to $100 \%$, positive predictive value $=100 \%$, negative predictive value $=96.6 \%(95 \%$ confidence interval $94.7 \%$ to $97.9 \%$ ), and accuracy $=97 \%$ (95\% confidence interval $92.6 \%$ to $99.2 \%)$. The McNemar test highlighted a difference of $10.3 \%$ between the FLUS and TUS. The agreement between the TUS and FLUS was substantial (weighted kappa test 0.78). Although FLUS shows some limitations in diagnosing lung lesions associated with BRDC compared to the systematic approach, this study shows that the focused method could be used as an additional tool for evaluating consolidation, especially when examining a large number of post-weaned dairy calves.

Key words: Bovine respiratory disease; Calves; Focused ultrasonography; Thoracic ultrasonography 
53 Thoracic ultrasonography (TUS) is a useful calf-side diagnostic tool for detecting lung lesions associated with bovine respiratory disease complex (BRDC) (Ollivett et al., 2015). There are several techniques for performing TUS in dairy calves which vary in terms of the number of intercostal spaces (ICS) evaluated and the interpretation of the positivity of the test based on the depth and extension of the observed lesions (Teixeira et al., 2017; Ollivett and Buczinski, 2016; Buczinski et al., 2018a; Ollivett et al., 2015; Cramer and Ollivett, 2019). Ollivett and Buczinski (2016) described an ultrasonographic 6-point scoring system based on the portion of lung tissue involved in 3 types of ultrasonographic lung lesions (i.e., comet tails, lobular and lobar pneumonia), identified by scanning the right lung from the tenth to the first ICS and the left lung from the tenth to the second ICS, each ICS characterized by a specific intra-thoracic anatomical landmark (Ollivett et al., 2015). Assessing the entire lung field on both sides of the thorax has proven useful (Buczinski et al., 2014) and enables the extent of lung lesions to be evaluated, thus facilitating the prognostic evaluation of the affected the cranial thorax in older, heavily muscled calves, thus preventing a complete ultrasonographic examination (Ollivett and Buczinski, 2016). The hypothetical added value of assessing the cranial part of the lung compared to the extra time needed was recently investigated (Berman et al., 2019). Although the study was conducted in a low prevalence context of the disease that may have altered the power to estimate sensitivity (Se) accurately, these authors found that the presence of a lung consolidation caudal to the heart, with a depth of $3 \mathrm{~cm}$ or more, yielded an excellent Se and specificity (Sp) (89\% and 95\%, respectively) in diagnosing pneumonia in preweaned calves using a latent class model (Berman et al., 2019).

Currently, there are no data on a simplified ultrasonographic technique that could both be used in preweaned and postweaned dairy calves with a mid to high prevalence of lung lesions. Our study therefore aimed to compare the results of focused lung ultrasonography (FLUS) for the diagnosis of lung lesions associated with BRDC in 1 to 6-month-old dairy calves to traditional systematic TUS 
used in preweaned dairy calves. The FLUS was performed by rapidly evaluating the lung lobes that are frequently involved in pneumonia and which are also the easiest to reach ultrasonographically in older calves, i.e., the caudal aspect of the cranial left lung lobe (fifth and fourth left ICS), the middle right lung lobe and the caudal aspect of the right cranial lung lobe, (fifth and fourth right ICS). We hypothesized that FLUS could be a clinically appropriate method for diagnosing lung lesions associated with BRDC and that there would be a good level of agreement with systematic TUS.

A prospective diagnostic accuracy study was performed according to the relevant standards (Bossuyt et al., 2015), using a convenience sample selected from dairy farms that were regularly checked by our ambulatory clinic from September 2018 to April 2019. Criteria for the selection of the farms were: a recent history of BRDC in at least 1 calf pen detected by the herd practitioner, location of the farms (no further than a 1-hour drive from the Veterinary Teaching Hospital), willingness to capture calves, and milking cows per farm ranging from 180 to 230 . We selected calves in pens on each farm only if at least 1 calf showed signs of BRDC. In brief, an experienced veterinarian (AB) examined the calves to see if any of them presented a spontaneous cough. Coughing calves were scored using the Wisconsin calf respiratory scoring chart (McGuirk, 2008). If 1 of the coughing calves reached a total respiratory score of 5 or more, it was considered as having BRDC. All calves within the pen were then considered eligible for the study, irrespective of their external clinical status. This selection was performed to ensure a minimum prevalence of lung lesions in the affected pen in order to optimize the sample size for both groups of calves (calves with and without lesions). The selected animals were both male and female Holstein Friesian calves aged between 1 and 6 months. A maximum of 10 calves were examined per pen. A randomization application (Randomizer, Darshan Institute of Engineering and Technology, Rajkot, India), run on an Android smartphone, was used when more than 10 calves were present. Calves were excluded if: they belonged to breeds other than Holstein Friesian, were older than 6 months, or had other concurrent diseases.

The publication of data was approved by the Ethical Committee of the University of Milan (approval number 47/2017, November 28, 2017). 
104 Enrolled calves were submitted to thoracic ultrasonography using a portable unit (Ibex Pro, EI 105 Medical) with a 7.5 MHz linear transducer designed for a transrectal purpose, set to a depth of $8 \mathrm{~cm}$ and gain of $16 \mathrm{~dB}$. Vegetable oil was sprayed over the thoracic skin (Brethour, 1992). The thorax was not shaved to reflect the rapid use in a field setting (Ollivett and Buczinski, 2016). FLUS was performed by an experienced veterinarian $(\mathrm{AB})$. The technique used was an abbreviation of the examination described in Ollivett and Buczinski 2016, based on the ventral landmarks described by

110 Ollivett et al. (2015). Focused ultrasonography of the left lung was performed first. The probe was 111 placed between the middle and dorsal third of the fifth ICS, immediately under the elbow. 112 Ultrasonography scanning was performed by moving the probe ventrally to the costochondral 113 junction $(\mathbf{C C J})$. The probe was then slipped cranially into the fourth ICS, starting between the middle 114 and dorsal third and moving again ventrally to the CCJ and pleural deviation. Similarly, on the right 115 site, the probe was placed over the fifth right ICS and then slipped cranially towards the fourth ICS to visualize the lung tissue until the heart was ventrally visualized. After performing FLUS on all calves in the same selected pen, systematic TUS (ICS 10-1 on the right, and ICS 10-2 on the left) was performed by the first author (DP) following the procedures 119 described by Ollivett and Buczinski (2016) by randomly re-catching all previously scanned calves. 120 This procedure was used to ensure blinding between FLUS and TUS examinations. Lung lesions 121 were scored directly on the farms. For both FLUS and TUS, we adopted the 6-point ultrasonographic scoring system described by Ollivett and Buczinski, (2016). A positive ultrasonographic examination test was defined when a calf had a TUS score of 2 or more (lobular pneumonia: consolidation $\geq 1 \mathrm{~cm}$ ) 124 (Ollivett and Buczinski, 2016). Since FLUS enables 3 lung lobes to be visualized (the caudal aspect 125 of the cranial left lung lobe [fifth and fourth left ICS], the middle right lung lobe and the caudal aspect 126 of the right cranial lung lobe, [fifth and fourth right ICS]), the whole 6-point scoring scale and the same cut-off point as the full technique were used. and analysis. Sex was reported as frequency and percentage of males and females. Age was not 
normally distributed and reported with median, interquartile range (IQR) from the 25th percentile to

131 the 75th percentile, minimum (min) and maximum (max) values. The TUS and FLUS scores were 132 reported as frequencies and percentages. Since the initial aim was to quantify the significance of 133 information loss due to FLUS vs. complete examination, we based our sample size calculation first 134 on a minimal agreement beyond the chance of reaching agreement between the 2 tests. Specifically, 135 we based our sample size calculation on an acceptable Kappa level of at least 0.80 , with a minimal 136 lower bound of 0.61 . The 0.61 level was determined a priori as the lower bound of strong agreement 137 between 2 different measures (Dohoo et al., 2009). Different scenarios were tested. Based on a sample 138 size with more than 120 calves would fit in most of the scenarios using type 1 error $5 \%$, type 2 error $13920 \%$. The accuracy of FLUS, Se, Sp, positive predictive value (PPV), and negative predictive value 140 (NPV) were calculated using TUS as the gold standard.

141 To investigate whether there was a systematic, significant difference in the number of cases resulting as positive between TUS and FLUS, the McNemar test was performed. In addition, the inter-test agreement between FLUS and TUS scores was assessed using the weighted kappa $\left(\boldsymbol{\kappa}_{\mathbf{w}}\right)$ (Dohoo et al., 2009). The clinical application of the differences between TUS and FLUS findings was further investigated, modelling various previous scenarios from 0 to 1 with 0.05 increment steps. The apparent disease prevalence based on FLUS findings was calculated based on the true prevalence of TUS positive cases and FLUS accuracy (Se and Sp). These were derived from the formula by Dohoo et al. (2009):

$$
\text { Apparent prevalence }=\operatorname{Prev}^{*} \mathrm{Se}+(1-\mathrm{Sp}) *(1-\operatorname{Prev})
$$

150 The uncertainty around the estimates was obtained using low and high $95 \%$ confidence intervals 151 limits of Se and Sp, respectively. A logistic regression model was also performed to underline the 152 possible effect of age and the interaction between age and FLUS on TUS results. Specifically, in the 153 logistic regression model, the outcome of interest was TUS (positive when a calf had a TUS score $\geq$ 1542 , negative with a score of $<2$ ), and the independent variables checked were the FLUS score, age, and 155 the interaction between FLUS and age, using backward stepwise regression. Age was used as a 
156 categorical variable and classified according to the median (i.e., lower than the median, and greater 157 than or equal to the median).

158 Eleven male (8.1\%) and 124 female calves (91.9\%) belonging to 10 dairy farms were enrolled in the 159 study with a total number of 135 calves. The median age of the calves was 84 (IQR $25 \% 54$ days; 160 IQR 75\% 115 days; min 30 days; max 183 days). Sixty-two calves (45.9\%) had a FLUS score of 2 161 or more. Seventy-six calves $(56.2 \%)$ had a TUS score of 2 or more and were therefore considered to 162 have pneumonia. For each FLUS and TUS scoring class, Table 1 summarizes the number of animals 163 and percentages, also split by the calf age. Using a TUS threshold score of $\geq 2$, FLUS had a Se of $16481.6 \%$ (95\% confidence interval [CI] 71\% to 89.5\%), a Sp of 100\% (95\% CI 93.9\% to 100\%), a PPV 165 of $100 \%$, an NPV of $96.6 \%$ (95\% CI $94.7 \%$ to $97.9 \%)$, and an accuracy of $97 \%(95 \%$ CI $92.6 \%$ to $166 \quad 99.2 \%)$.

167 Figure 1 shows the impact of the simulated true prevalence of calves with TUS lesions on the apparent 168 prevalence using FLUS. The impact of using FLUS for prevalence assessment vs. TUS as a gold 169 standard revealed that the difference between the 2 methods was minimal in prevalence settings lower 170 than 30 to $50 \%$. A comparison between FLUS and TUS in the current setting revealed 10 false 171 negatives with lesions detected by TUS. Of these false negatives, eight calves were affected by lobular 172 consolidation in the cranial aspect of the right or left cranial lung lobes, while 2 calves showed a 173 consolidation of the entire cranial aspect of the right cranial lung lobe. None of the 10 false-negative 174 cases showed lesions in other lung lobes. There were no false positives when using FLUS. The 175 McNemar test underlined a difference of $10.37 \%$ between FLUS and TUS ( $\mathrm{P}=0.001 ; 95 \%$ CI $5.23 \%$ 176 to $15.51 \%)$. The agreement between FLUS and TUS, calculated using the $\kappa_{\mathrm{w}}$ test, was substantial $\left(\kappa_{\mathrm{w}}\right.$ $1770.778 ; 95 \%$ CI 0.718 to 0.837 ). The comparison between FLUS and TUS scores is shown in Table 2. 178 According to the applied logistic regression model, age did not influence the relationship between 179 FLUS and TUS $(\mathrm{P}=0.453)$. Furthermore, the interaction between age and FLUS did not influence the 180 TUS results $(\mathrm{P}=1.00)$. 
181 The results of this study were expected because the protocol chosen for FLUS was based on the

182 literature that evaluated the topographical distribution of lung abnormalities in suppurative 183 bronchopneumonia, which is the most common form of pneumonia in young dairy calves (Panciera 184 and Confer, 2010). These results reflect those of Berman et al. (2019), who found that the diagnostic 185 accuracy indexes obtained with the scan of the caudal part of the lung (eleventh - third ICS right and 186 left) alone were satisfactory compared to those obtained by ultrasonographically scanning the full 187 entire lung fields. Moreover, as Ollivett and Buczinski (2016) reported and confirmed by our results, 188 the lung lobes caudal to the fifth ICS are only rarely involved in pneumonia, and when they are, they are associated with more substantial cranial lesions. Although the results mentioned above were satisfactory, a note of caution is warranted for at least 2 reasons. Eight out of 10 false-negative calves using FLUS had lobular lesions cranially to the fourth ICS on the left or the right side of the thorax. Lobular pneumonia can be associated with viral infections and bacteria such as Pasteurella multocida or Mycoplasma bovis, which are responsible for lobular necrotic pneumonia or bronchiole-centered lesions with a progressive spread along with the surrounding tissue (Panciera and Confer, 2010). In addition, 2 out of 10 false negatives had deep consolidation of the cranial lung lobes. Although consolidation in these areas may also be due to fibrosis and atelectasis not directly correlated with BRDC pathogens (Berman et al., 2019), pneumonia cannot be ruled out in these false-negative cases because typically, lung lesions generally start in the cranial aspect of the right cranial lung lobe (accessible by scanning first and second ICS) with a subsequent caudal progression (Dagleish et al., 2010). Furthermore, Ollivett et al. (2015) demonstrated that lobar lesions of the right cranial lobe are consistent with bacterial pneumonia based on histological examination of the lung tissue. In our opinion, these results illustrate the intrinsic limitation of focused ultrasound techniques, which are not $100 \%$ accurate and cannot fully replace systematic ultrasonography.

The McNemar test substantiated this observation and showed a significant discrepancy between cases testing positive/negative at FLUS and TUS, respectively. Another source of uncertainty is related to the variation in the FLUS accuracy based on the prevalence of the disease. As expected, in the 
presence of a high Sp and imperfect Se, the FLUS examination could be used as a good alternative to TUS when the prevalence of lung lesion is not too high; e.g., $<30-50 \%$. However, this was based on simulations of different prevalence contexts, assuming a constant accuracy of FLUS across these ranges. In scenarios with a high prevalence of lung lesions, an underestimation associated with FLUS

211 is likely to be clinically relevant, highlighting the need to add the right cranial part of the right cranial 212 lung in the scanning, especially when it can be easily done (in calves less than 2 months old).

213 Although an in-depth evaluation of the impact of age on the diagnostic accuracy of FLUS was beyond 214 the scope of the current study, we found that age did not influence the FLUS results in this case study group. This supports the hypothesis that the loss of information due to the FLUS technique did not depend on age but probably on the technique. The use of FLUS could thus be recommended in diagnosing pneumonia in larger calves, when reaching the cranial fields of the thorax is difficult due

219 TUS should be preferred to FLUS in smaller calves in order to prevent the loss of valuable information (e.g., the early stage of the disease which commonly affects most cranial lobes). In addition, TUS should be preferred in high-risk herds, considering that the full technique does not present any limitation in these animals and highlights the extent of the consolidation, thus representing more useful information in terms of prognostic factors.

The main limitation of this study was that FLUS was compared to TUS, which itself is an imperfect gold standard. Although it is important to consider the possible bias of this technique, the detection of lobar or lobular lesions by systematic TUS is the most accurate in vivo diagnostic tool. 227 Furthermore, because FLUS and TUS are conditionally dependent, the covariance between the 2 tests 228 could lead to the same classification error. Nonetheless, FLUS can help the clinician and can be used 229 for the early diagnosis of lung lesions associated with BRDC in older animals because it is reasonably 230 accurate, easy and rapid to perform. Two different clinicians performed TUS and FLUS, respectively. 231 This could be perceived as a limitation of the study. However, it was difficult to guarantee that a 232 specific clinician performing the 2 tests would remain blind to the first examination results (therefore 
233 inducing a risk of recall bias falsely inflating the test accuracy). We therefore preferred to use 2 234 experienced clinicians to perform the examination. Including a gap between the 2 examinations 235 performed by the same clinician could also have been a solution. However, there would have been a 236 risk of lesion progression between the 2 examinations as previously reported (Ollivett and Buczinski, 237 2016), which would also have introduced a risk of bias. Due to the high level of experience of both 238 ultrasonographers, we are confident that using 2 clinicians was the best way to limit the current risk 239 of bias.

240 A further potential limitation affecting the differences observed between the FLUS and TUS could 241 have been a disagreement in interpreting the lung lesions between the 2 clinicians performing the 242 examinations. Although the discrepancy could have been assessed using an agreement study between 243 the 2 operators, inter-rater agreement for the lung ultrasonography is generally good for evaluating 244 lung consolidation, comet tails, and pleural effusion (Buczinski et al., 2018b). Another limitation of 245 the current study is the lack of investigations on the etiological agents involved. This information 246 would have been very useful to correlate the lobular lesions to a specific etiological agent, especially 247 in the FLUS false-negative calves. Further studies are therefore warranted to investigate this topic. 248 The results of this study have various clinical implications. An important finding is that FLUS 249 represents a practical method to diagnose lung lesions in field conditions and can be performed 250 rapidly using a linear probe. Focused lung ultrasonography may help practitioners to recognize lung 251 lesions in post-weaned calves. The easy recognition of sick animals would facilitate specific 252 management vs. healthy subjects such as early separation, thereby preventing unnecessary 253 antimicrobial treatments of healthy cohabitants and spreading pathogens among the groups. In 254 conclusion, we believe that FLUS is a promising tool to improve the diagnosis of pneumonia in older 255 calves where there is a limited access to the cranial part of the thorax. 
258 Berman J., D. Francoz, S. Dufour, S. Buczinski. 2019. Bayesian estimation of sensitivity and specificity of systematic thoracic ultrasound exam for diagnosis of bovine respiratory disease in pre-weaned calves. Prev Vet Med. 162:38-45. https://doi.org/10.1016/j.prevetmed.2018.10.025

Bossuyt, P. M., J. B. Reitsma, D. E. Bruns, C. A. Gatsonis, P. P. Glasziou, L. Irwig, J. G. Lijmer, D. Moher, D. Rennie, H. C. de Vet, H. Y. Kressel, N. Rifai, R. M. Golub, D. G. Altman, L. Hooft, D. A. Korevaar, J. F. Cohen, and STARD Group. 2015. STARD 2015: An Updated List of Essential Items for Reporting Diagnostic Accuracy Studies. Radiology. 277(3):826-832. https://doi.org/10.1148/radiol.2015151516

Brethour J.R. 1992. The repeatability and accuracy of ultrasound in measuring backfat of cattle. J Anim Sci. 70(4):1039-1044. https://doi.org/10.2527/1992.7041039x

Buczinski S., C. Faure, S. Jolivet, A. Abdallah. 2016. Evaluation of inter-observer agreement when using a clinical respiratory scoring system in pre-weaned dairy calves. N Z Vet J. 64(4):243247. https://doi.org/10.1080/00480169.2016.1153439

Buczinski S., G. Forté, D. Francoz, A. M. Bélanger. 2014. Comparison of thoracic auscultation, clinical score, and ultrasonography as indicators of bovine respiratory disease in preweaned dairy calves. J Vet Intern Med. 2014;28(1):234-242. https://doi.org/10.1111/jvim.12251

Buczinski S., M.E. Borris, J. Dubuc. 2018a. Herd-level prevalence of the ultrasonographic lung lesions associated with bovine respiratory disease and related environmental risk factors. $\mathrm{J}$ Dairy Sci. 101(3):2423-2432. https://doi.org/10.3168/jds.2017-13459

Buczinski, S., C. Buathier, A. M. Bélanger, H. Michaux, N. Tison, E. Timsit. 2018b. Inter-rater agreement and reliability of thoracic ultrasonographic findings in feedlot calves, with or without naturally occurring bronchopneumonia. Journal of veterinary internal medicine, 32(5), 1787 1792. https://doi.org/10.1111/jvim.15257 
282 Cramer, M. C., and T. L. Ollivett. 2019. Growth of preweaned, group-housed dairy calves diagnosed with respiratory disease using clinical respiratory scoring and thoracic ultrasound-A cohort study. J Dairy Sci. 102(5):4322-4331. https://doi.org/10.3168/jds.2018-15420

Dagleish, M. P., Finlayson, J., Bayne, C., MacDonald, S., Sales, J., \& Hodgson, J. C. (2010). Characterization and time course of pulmonary lesions in calves after intratracheal infection

with Pasteurella multocida A: 3. Journal of comparative pathology, 142(2-3), 157-169. https://doi.org/10.1016/j.jcpa.2009.10.015

Dohoo, I., W. Martin, andH. Stryhn.2003. Veterinary Epidemiologic Research. AVC Inc., Charlottetown, PEI, Canada.

McGuirk S. M. 2008. Disease management of dairy calves and heifers. Vet Clin North Am Food Anim Pract. 24:139-153. https://doi.org/10.1016/j.cvfa.2007.10.003

Ollivett, T. L., J. L. Caswell, D. V. Nydam, T. Duffield, K. E. Leslie, J. Hewson, and D. Kelton. 2015. Thoracic Ultrasonography and Bronchoalveolar Lavage Fluid Analysis in Holstein Calves with Subclinical Lung Lesions. J Vet Intern Med. 29(6):1728-1734. https://doi.org/10.1111/jvim.13605

Panciera, R. J., and A. W. Confer. 2010. Pathogenesis and pathology of bovine pneumonia. Vet Clin North Am Food Anim Pract. 26(2):191-214. https://doi.org/10.1016/j.cvfa.2010.04.001

Ollivett T. L. and S. Buczinski. 2016. On-farm use of ultrasonography for bovine respiratory disease. Vet Clin North Am Food Anim Pract. 32:19-35. https://doi.org/10.1016/j.cvfa.2015.09.001

Teixeira, A., J. McArt, and R. C. Bicalho. 2017. Thoracic ultrasound assessment of lung consolidation at weaning in Holstein dairy heifers: Reproductive performance and survival. J Dairy Sci. 100(4):2985-2991. https://doi.org/10.3168/jds.2016-12016 


\section{Table 1}

305 Ultrasonography scores used to classify the severity of lung lesions associated with bovine respiratory 306 disease complex for 2 different methods of lung ultrasonography (Focused Lung Ultrasonography 307 [FLUS], and Thoracic Ultrasonography [TUS]) in 135 Holstein Friesian calves from 10 dairy herds 308 in Italy. Columns report animals grouped according to the TUS score and the binary interpretation of 309 the same score (i.e., animals testing positive vs. animal testing negative). Rows report total numbers 310 of animals as well as grouped according to age. For both FLUS and TUS, the 6-point ultrasonographic 311 scoring system described by Ollivett and Buczinski (2016) was applied: calves with a TUS score of 3120 or 1 were considered healthy, while calves with a TUS score of 2 (lobular consolidation), 3 313 (consolidation of 1 lung lobe), 4 (consolidation of 2 lung lobes), or 5 (consolidation of 3 or more lung 314 lobes) were considered to be affected by bovine respiratory disease.

\begin{tabular}{|c|c|c|c|c|c|c|c|c|c|}
\hline & $\begin{array}{l}\text { TUS } \\
\text { Score }\end{array}$ & 0 & 1 & 2 & 3 & 4 & 5 & $\begin{array}{l}\text { Tested } \\
\text { negative }\end{array}$ & $\begin{array}{l}\text { Tested } \\
\text { positive }\end{array}$ \\
\hline \multirow{2}{*}{ Total cases } & LUS & $\mathrm{z}$ & $\begin{array}{l}22 \\
(16.3 \%)\end{array}$ & $\begin{array}{l}19 \\
(14.1 \%)\end{array}$ & $\begin{array}{l}14 \\
(10.4 \%)\end{array}$ & $\begin{array}{l}13 \\
(9.6 \%)\end{array}$ & $\begin{array}{l}16 \\
(11.9 \%)\end{array}$ & $\begin{array}{l}73 \\
(54.1 \%)\end{array}$ & $\begin{array}{l}62 \\
(45.9 \%)\end{array}$ \\
\hline & TUS & $\begin{array}{l}38 \\
(28.1 \%)\end{array}$ & $\begin{array}{l}21 \\
(15.6 \%)\end{array}$ & $\begin{array}{l}25 \\
(18.5 \%)\end{array}$ & $\begin{array}{l}11 \\
(8.1 \%)\end{array}$ & $\begin{array}{l}12 \\
(8.9 \%)\end{array}$ & $\begin{array}{l}28 \\
(20.7 \%)\end{array}$ & $\begin{array}{l}59 \\
(43.7 \%)\end{array}$ & $\begin{array}{l}76 \\
(56.3 \%)\end{array}$ \\
\hline \multirow{2}{*}{$\begin{array}{l}\text { Calves } 1 \\
\text { month old } \\
(\mathrm{n} .=13)\end{array}$} & FLUS & $\begin{array}{l}2 \\
(15 .\end{array}$ & $\begin{array}{l}4 \\
(30.8 \%)\end{array}$ & $\begin{array}{l}4 \\
(30.8 \%)\end{array}$ & I & $\begin{array}{l}2 \\
(15.4 \%)\end{array}$ & $\begin{array}{l}1 \\
(7.7 \%)\end{array}$ & $\begin{array}{l}6 \\
(46.2 \%)\end{array}$ & $\begin{array}{l}7 \\
(53.8 \%)\end{array}$ \\
\hline & TUS & $\begin{array}{l}2 \\
(15.4 \%)\end{array}$ & $\begin{array}{l}3 \\
(23.1 \%)\end{array}$ & $\begin{array}{l}4 \\
(30.8 \%)\end{array}$ & $\begin{array}{l}1 \\
(7.7 \%)\end{array}$ & $\begin{array}{l}2 \\
(15.4 \%)\end{array}$ & $\begin{array}{l}1 \\
(7.7\end{array}$ & $\begin{array}{l}5 \\
(38.5 \%)\end{array}$ & $\begin{array}{l}8 \\
(61.5 \%)\end{array}$ \\
\hline \multirow{2}{*}{$\begin{array}{l}\text { Calves } 2- \\
\text { months-old } \\
(\mathrm{n} .=48)\end{array}$} & FLUS & $\begin{array}{l}17 \\
(35.4 \%)\end{array}$ & $\begin{array}{l}7 \\
(14.6 \%)\end{array}$ & $\begin{array}{l}6 \\
(12.5 \%)\end{array}$ & $\begin{array}{l}10 \\
(20.8 \%)\end{array}$ & $\begin{array}{l}4 \\
(8.3 \%)\end{array}$ & 4 & $\begin{array}{l}24 \\
(50.0 \%)\end{array}$ & $\begin{array}{l}24 \\
(50.0 \%)\end{array}$ \\
\hline & TUS & $\begin{array}{l}13 \\
(27.1 \%)\end{array}$ & $\begin{array}{l}5 \\
(10 \\
\end{array}$ & $\begin{array}{l}8 \\
(16.7 \%)\end{array}$ & $\begin{array}{l}6 \\
(12.5 \%)\end{array}$ & $\begin{array}{l}5 \\
(10.4 \%) \\
\end{array}$ & $.9 \%)$ & $\begin{array}{l}18 \\
(37.5 \%)\end{array}$ & $\begin{array}{l}30 \\
(62.5 \%)\end{array}$ \\
\hline \multirow{2}{*}{$\begin{array}{l}\text { Calves } 3- \\
\text { month-old } \\
(\mathrm{n} .=28)\end{array}$} & $\mathrm{F}$ & (3 & (1 & $\begin{array}{l}4 \\
(14.3 \%)\end{array}$ & $\begin{array}{l}3 \\
(10.7 \%)\end{array}$ & $\begin{array}{l}1 \\
(3.6 \%)\end{array}$ & $\begin{array}{l}4 \\
(14 .\end{array}$ & $\begin{array}{l}16 \\
(57.1 \%)\end{array}$ & $\begin{array}{l}12 \\
(42.9 \\
\%)\end{array}$ \\
\hline & TUS & $\begin{array}{l}7 \\
(25.0 \%)\end{array}$ & $\begin{array}{l}7 \\
(25.0 \%)\end{array}$ & $\begin{array}{l}5 \\
(17.9 \%) \\
\end{array}$ & $\begin{array}{l}2 \\
(7.1 \%)\end{array}$ & $\begin{array}{l}3 \\
(10.7 \%)\end{array}$ & $\begin{array}{l}4 \\
(14.3 \%)\end{array}$ & $\begin{array}{l}14 \\
(50.0 \%)\end{array}$ & $\begin{array}{l}14 \\
(50.0 \%)\end{array}$ \\
\hline \multirow{2}{*}{$\begin{array}{l}\text { Calves } 4- \\
\text { month-old } \\
(n .=26)\end{array}$} & $\mathrm{F}$ & $\begin{array}{l}11 \\
(42.3 \%)\end{array}$ & $\begin{array}{l}3 \\
(11.5 \%)\end{array}$ & $\begin{array}{l}3 \\
(11.5 \%)\end{array}$ & l & $\begin{array}{l}4 \\
(15.4 \%)\end{array}$ & $\begin{array}{l}5 \\
(19.2 \%)\end{array}$ & $\begin{array}{l}14(53.8 \\
\%)\end{array}$ & $\begin{array}{l}12 \\
(46.2 \%)\end{array}$ \\
\hline & TUS & $\begin{array}{l}7 \\
(26.9 \%)\end{array}$ & $\begin{array}{l}4 \\
(15.4 \%)\end{array}$ & $\begin{array}{l}5 \\
(19.2 \%)\end{array}$ & / & $\begin{array}{l}2 \\
(7.7 \%)\end{array}$ & $\begin{array}{l}8 \\
(30.8 \%)\end{array}$ & $\begin{array}{l}11(42.3 \\
\%)\end{array}$ & $\begin{array}{l}15 \\
(57.7 \%)\end{array}$ \\
\hline \multirow{2}{*}{$\begin{array}{l}\text { Calves 5- } \\
\text { month-old } \\
(\mathrm{n} .=17)\end{array}$} & FLUS & $\begin{array}{l}8 \\
(47 .\end{array}$ & $\begin{array}{l}2 \\
(11 .\end{array}$ & $\begin{array}{l}2 \\
(11.8 \%)\end{array}$ & $\begin{array}{l}1 \\
(5.9 \%)\end{array}$ & $\begin{array}{l}2 \\
(11.8 \%)\end{array}$ & $\begin{array}{l}2 \\
(11.8 \%)\end{array}$ & $\begin{array}{l}10(58.8 \\
\%)\end{array}$ & $\begin{array}{l}7 \\
(41.2 \%)\end{array}$ \\
\hline & TUS & $\begin{array}{l}7 \\
(41.2 \%) \\
\end{array}$ & $\begin{array}{l}1 \\
(5.9 \%) \\
\end{array}$ & $\begin{array}{l}3 \\
(17.6 \%) \\
\end{array}$ & $\begin{array}{l}2 \\
(11.8 \%)\end{array}$ & 1 & $\begin{array}{l}4 \\
(23.5 \%)\end{array}$ & $\begin{array}{l}8 \\
(47.1 \%)\end{array}$ & $\begin{array}{l}9 \\
(52.9 \%)\end{array}$ \\
\hline \multirow{2}{*}{$\begin{array}{l}\text { Calves 6- } \\
\text { month-old } \\
(\mathrm{n} .=3)\end{array}$} & FLUS & $\begin{array}{l}2 \\
(66.7 \%)\end{array}$ & $\begin{array}{l}1 \\
(33.3 \%)\end{array}$ & / & / & / & / & $\begin{array}{l}3 \\
(100 \%)\end{array}$ & 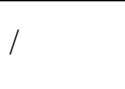 \\
\hline & TUS & $\begin{array}{l}2 \\
(66.7 \%)\end{array}$ & $\begin{array}{l}1 \\
(33.3 \%)\end{array}$ & I & / & / & 1 & $\begin{array}{l}3 \\
(100 \%)\end{array}$ & / \\
\hline
\end{tabular}




\section{Table 2}

317 Contingency table and weighted kappa $\left(\kappa_{\mathrm{w}}\right)$ value for the comparison between focused lung

318 ultrasonography (FLUS) scores and thoracic ultrasonography (TUS) scores.

\begin{tabular}{l|l|llllll|l|l}
\hline \multicolumn{10}{c}{ FLUS } \\
\hline \multirow{1}{*}{ TUS } & Scores $^{\mathrm{a}}$ & 0 & 1 & 2 & 3 & 4 & 5 & Number of calves \\
\cline { 2 - 9 } & 0 & 38 & 0 & 0 & 0 & 0 & 0 & 38 \\
& 1 & 8 & 13 & 0 & 0 & 0 & 0 & 21 \\
& 2 & 4 & 6 & 15 & 0 & 0 & 0 & 25 \\
& 3 & 0 & 3 & 2 & 5 & 1 & 0 & 11 \\
& 4 & 1 & 0 & 2 & 6 & 2 & 1 & 12 \\
& 5 & 0 & 0 & 0 & 3 & 10 & 15 & 28 \\
\cline { 2 - 8 } & Number of calves & 51 & 22 & 19 & 14 & 13 & 16 & 135 \\
\hline
\end{tabular}

320 The weighted Kappa coefficient was 0.78 (95\% CI: 0.72 to 0.84 ) indicating a substantial agreement (Dohoo et al., 2009 ).

321 a For both FLUS and TUS, the 6-point ultrasonographic scoring system described by Ollivett and Buczinski (2016) was 322 applied: calves with a TUS score of 0 or 1 were considered healthy, while calves with a TUS score of 2 (lobular 323 consolidation), 3 (consolidation of 1 lung lobe), 4 (consolidation of 2 lung lobes) or 5 (consolidation of 3 or more lung 324 lobes) were considered to be affected by bovine respiratory disease. 
325 Figure 1. Apparent prevalence of lung lesions using focused lung ultrasonography (FLUS) (with a consolidation threshold of $\geq 1 \mathrm{~cm}$ within a normally aerated lung lobe; score 2 ) according to different disease prevalence scenarios of lung lesions using thoracic ultrasonography (TUS) as a gold standard.

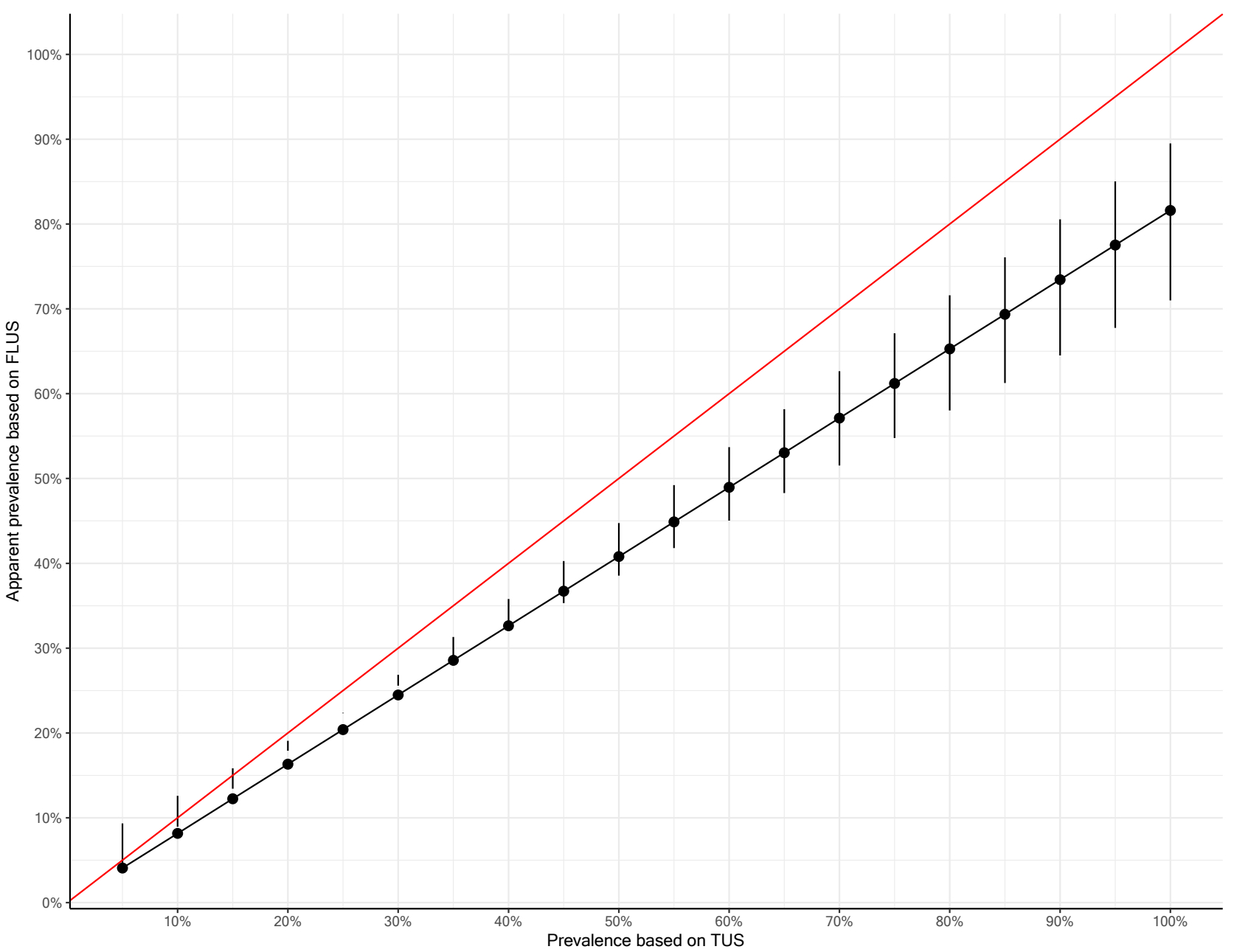

This figure depicts the relation between apparent prevalence based on FLUS vs. the true prevalence of lung lesions when assessed by TUS using the observed FLUS sensitivity $(81.6 \%, 95 \% \mathrm{CI}(71.0-89.5))$ and specificity $100 \%(93.9-100 \%)$ based on various simulated ranges of lung lesion prevalence (from 0 to 1 by 0.05 increment steps). The black line and associated dots represent the mean estimate. The upper and lower bounds around each specific dot are based on the apparent prevalence calculation using low and high Se and Sp bounds, respectively. The identity line $(\mathrm{y}=\mathrm{x})$ is indicated in red. The green shaded area depicts the prevalence range where TUS and FLUS prevalence estimations are less than $5 \%$. The red area depicts an underestimation by FLUS of $10 \%$ or less of the real prevalence ranges. The grey shaded area depicts an underestimation by FLUS of between 5 and $10 \%$ of the true prevalence. 
